\title{
ATUAÇÃO DOS PROFISSIONAIS DA INFORMAÇÃO NO CICLO DE VIDA DOS DADOS - DATAONE: UM ESTUDO COMPARADO
}

\author{
INFORMATION PROFESSIONALS PERFORMANCE IN \\ THE DATA LIFE CYCLE - DATAONE: A COMPARED \\ STUDY
}

Renata Lemos dos Anjosa

Guilherme Ataíde Dias ${ }^{b}$

\begin{abstract}
RESUMO
Introdução: com a consolidação das Tecnologias Digitais de Informação e Comunicação na vasta maioria dos processos humanos no início do século XXI, diariamente, produzimos uma infinidade de dados. Essa avalanche de dados é denominada como o fenômeno Big Data. Na seara acadêmica, novas possibilidades relacionadas ao uso de dados de pesquisas, estão emergindo. Pesquisadores das mais variadas áreas estão dando especial atenção ao Plano de Gestão de Dados, o qual envolve a curadoria dos dados de pesquisa, durante todo o seu ciclo de vida, desde o seu planejamento até o seu (re)uso. Objetivo: busca investigar como os profissionais da informação podem atuar nas etapas componentes do Ciclo de Vida dos Dados DataONE. Metodologia: configura-se como uma pesquisa exploratória-descritiva, e bibliográfica, assumindo o método comparado o qual associou as competências dos profissionais da informação que podem ser aplicadas e/ou adaptadas na realização das etapas componentes do Ciclo de Vida dos Dados - DataONE. Resultados: verificou-se que a maioria das competências dos profissionais da informação alinham-se às componentes do ciclo de vida dos dados. Conclusões: concluímos que as competências dos profissionais da informação podem ser efetivamente aplicadas e adequadas à realidade dos dados de pesquisa, mais precisamente no processo do ciclo de vida dos dados.
\end{abstract}

Descritores: Dados científicos. Profissional da informação. Ciclo de vida dos dados. Dataone.

\footnotetext{
a Mestranda no Programa de Pós-Graduação em Ciência da Informação da Universidade Federal da Paraíba (PPGCI-UFPB). E-mail: renatalemosdosanjos@gmail.com

b Professor nos Programas de Pós-Graduação em Ciência da Informação - PPGCl e no Programa de Pós-Graduação em Gestão nas Organizações Aprendentes - MPGOA, ambos na Universidade Federal da Paraíba (UFPB). E-mail: guilhermeataide@gmail.com
} 


\section{INTRODUÇÃO}

Com a inserção das Tecnologias Digitais de Informação e Comunicação (TDICs) em praticamente todos os processos humanos no início do século XXI, diariamente, mesmo sem percebermos, estamos produzindo uma avalanche de dados, por meio da internet das coisas (Internet of Things - loT), dos nossos smartphones, tablets, notebooks, GPS, e diversos outros dispositivos digitais. Esse dilúvio de dados é conhecido como o fenômeno Big Data, que se caracteriza como a rápida geração e processamento de uma grande quantidade de dados heterogêneos disponíveis digitalmente (CONEGLIAN; SEGUNDO; SANT'ANA, 2017).

Nessa perspectiva, McAffe e Brynjolfsson (2012) relacionam três características fundamentais pertinentes ao Big Data, denominadas os três Vs, a saber: volume, variedade e velocidade. Volume está relacionado a quantidade avassaladora de dados que são gerados diariamente. Variedade é correlacionada à diversidade, heterogeneidade desses dados. E velocidade em relação à rapidez em que esses dados são disponibilizados na rede em tempo real.

Os dados são um realidade emergente, de acordo com uma publicação recente do periódico The Economist, "o recurso mais valioso do mundo já não é petróleo, e sim dados ${ }^{1}$ " (2017, online, tradução nossa). Nesta mesma publicação, a The Economist afirma que as empresas gigantes da tecnologia são assim denominadas por possuírem um elevadíssimo número de dados, que são alvo de interesse de empresas, organizações, governos, entidades acadêmicas, entre outros, para serem utilizados visando os mais diversos fins.

$\mathrm{Na}$ esfera acadêmica, novas possibilidades relacionadas ao uso de dados provenientes de investigações científicas, dados de pesquisas, estão emergindo (BORGMAN, 2015), por configurar-se como fontes primárias de informação, e também, como subsídios para estudos futuros. Em concordância, Sayão e Sales (2013, p. 3) afirmam que "os pesquisadores, as instituições acadêmicas e as agências de fomento à pesquisa começam a compreender que estes dados, se

1 The world's most valuable resource is no longer oil, but data.

Inf. Inf., Londrina, v. 24, n. 1, p. $80-101$, jan./abr. 2019. 
devidamente tratados, preservados e gerenciados, podem constituir uma fonte inestimável de recursos informacionais". Em síntese, a preservação e o compartilhamento de dados caracterizam uma nova forma de se fazer ciência, denominada e-Science. Gray (2005 apud SALES et al., 2013) introduz o conceito de e-Science, definindo-a como a "ciência é produzida a partir do uso, processamento, análise e compartilhamento de dados de pesquisa", ou seja, a e-Science caracteriza-se por ser uma ciência voltada, orientada por dados.

Nesta perspectiva, pesquisadores das mais variadas áreas estão dando especial atenção aos seus dados, bem como ao Data Management Plan (DMP), ou ao Plano de Gestão de Dados (PGD), que em síntese "[...] envolve a gestão de dados de pesquisa desde a sua gênese, assegurando a preservação por longo prazo, descoberta, interpretação e reuso" (SAYÃO; SALES, 2012, p. 179), ou seja, envolve processos relacionados com a curadoria dos dados de pesquisa, durante todo o seu ciclo de vida.

No tocante ao ciclo de vida dos dados, este configura-se como um instrumento que auxilia os pesquisadores em vários estágios do processo das suas investigações científicas. As ações associadas a cada etapa do ciclo de vida dos dados são fundamentais para o processo de curadoria, garantindo uso otimizado e estendido aos dados. Dentre os mais variados modelos de ciclo de vida dos dados encontrados na literatura da área, Sayão e Sales (2015) destacam os seguintes: Curation Lifecycle Model proposto pelo Digital Curation Centre (DCC) $)^{2}$; O DDI Combined Lifecycle model elaborado pela Data Documentation Initiative (DDI) ${ }^{3}$; o Research Data Lifecycle proposto pela UK Data Service4; e o Data Lifecycle concebido pela Data Observation Network for Earth (DataONE) ${ }^{5}$, este, selecionado para fins deste estudo. No Brasil, temos nesta seara o ciclo de vida dos dados para a Ciência da Informação (CVD-CI) proposto por Sant'Ana (2016).

No âmbito da Ciência da Informação, a e-Science nos traz alguns questionamentos e desafios como, qual o papel dos profissionais da informação,

\footnotetext{
2 http://www.dcc.ac.uk/resources/curation-lifecycle-model

${ }^{3} \mathrm{http}: / / \mathrm{www}$.ddialliance.org/Specification/DDI-Lifecycle/

${ }^{4} \mathrm{http}: / / \mathrm{www}$.data-archive.ac.uk/create-manage/life-cycle

${ }^{5} \mathrm{https}: / / \mathrm{www}$.dataone.org/data-life-cycle
}

Inf. Inf., Londrina, v. 24, n. 1, p. 80 -101, jan./abr. 2019. 
no tratamento dos dados primários oriundos de investigações científicas, e como os mesmos podem atuar efetivamente no ciclo de vida dos dados.

Nesse contexto, Costa e Cunha (2014, p. 194) afirmam que "os dados produzidos pela e-Science trazem impactos profundos sobre a ciência e, portanto, exigem um exame acurado das funções das instituições empenhadas no avanço da ciência e no apoio aos cientistas, dentre elas as bibliotecas". De uma forma geral, tanto as bibliotecas, quanto os seus profissionais precisam apoiar a e-Science.

Burton e Lyon (2017, online, tradução nossa) afirmam que a Associaton of College e Research Libraries (ACRL, 2015):

[...] identificou a necessidade de serviços de curadoria de dados mais avançados, instando os bibliotecários a ter um conhecimento mais aprofundado das práticas de pesquisa de domínio para permitir que eles ajudem os pesquisadores com gerenciamento, compartilhamento e preservação de dados ${ }^{6}$.

Fundamentado no que foi brevemente exposto, esta pesquisa investiga como os profissionais da informação podem atuar de forma efetiva em conjunto com a comunidade de pesquisadores no processo de curadoria dos dados, especificamente no modelo de ciclo de vida dos dados proposto pelo DataONE denominado Data Lifecycle, aqui mencionado doravante como Ciclo de Vida dos Dados - DataONE.

No que concerne ao objetivo geral, esta pesquisa busca investigar como os profissionais da informação podem atuar nas etapas componentes do Ciclo de Vida dos Dados - DataONE. Subsequente, delineou-se os seguintes objetivos específicos: a) descrever as etapas componentes do Ciclo de Vida dos Dados DataONE; b) identificar as competências dos profissionais da informação que podem ser adaptadas e/ou aplicadas na realização das etapas componentes do Ciclo de Vida dos Dados - DataONE e; c) elaborar quadro relacionando as competências dos profissionais da informação que podem ser adaptadas e/ou aplicadas na realização das etapas componentes do Ciclo de Vida dos Dados DataONE.

\footnotetext{
${ }^{6}(\ldots)$ identified a need for more advanced data curation services, urging librarians to have a deeper knowledge of domain research practices to enable them to help researchers with data management, sharing and preservation.
}

Inf. Inf., Londrina, v. 24, n. 1, p. $80-101$, jan./abr. 2019. 
Para atingir os objetivos propostos, a presente pesquisa configura-se como uma pesquisa exploratória e descritiva. Exploratória visando maior ampliação do entendimento acerca do assunto estudado. E descritiva, visando a descrição minuciosa das etapas componentes do Ciclo de Vida dos Dados DataONE, e das competências profissionais dos profissionais da informação propostas por Abels et al. (2003). E bibliográfica pela busca por materiais que abordassem as competências dos profissionais da informação.

Assume o método comparado, que é utilizando com a finalidade de verificar convergências e divergências, podendo apontar vínculos entre os fatores estudados (MARCONI; LAKATOS, 2000). No caso, objetivando a construção dos quadros para comparação, os quais associarão as competências dos profissionais da informação que podem ser adaptadas e/ou aplicadas na realização das etapas componentes do Ciclo de Vida dos Dados - DataONE, por meio da inferência dos conteúdos inseridos em ambos processos.

\section{CICLO DE VIDA DOS DADOS - DATAONE}

O Ciclo de Vida dos Dados - Data Lifecycle, conforme indicado anteriormente, é proposto pela iniciativa DataONE, que tem como missão "permitir a criação de novas ciências e conhecimento através do acesso universal aos dados sobre a vida na terra e o ambiente que a sustenta ${ }^{7}$ " (DataONE, online, tradução nossa).

Ainda em concordância com o DataONE (online, tradução nossa), o seu ciclo de vida dos dados fornece uma visão geral das etapas envolvidas para o gerenciamento e preservação de dados, visando posterior uso e reutilização. Ressalta-se que existem variações do ciclo de vida dos dados de acordo com práticas, domínios e necessidades de cada comunidade.

O ciclo de vida dos dados - DataONE é composto por oito etapas. A Figura 1, a seguir, ilustra como o mesmo está estruturado:

\footnotetext{
${ }^{7}$ Enable new science and knowledge creation through universal access to data about life on earth and the environment that sustains it.
}

Inf. Inf., Londrina, v. 24, n. 1, p. 80 - 101, jan./abr. 2019. 
Figura 1 - Ciclo de vida dos dados - DataONE

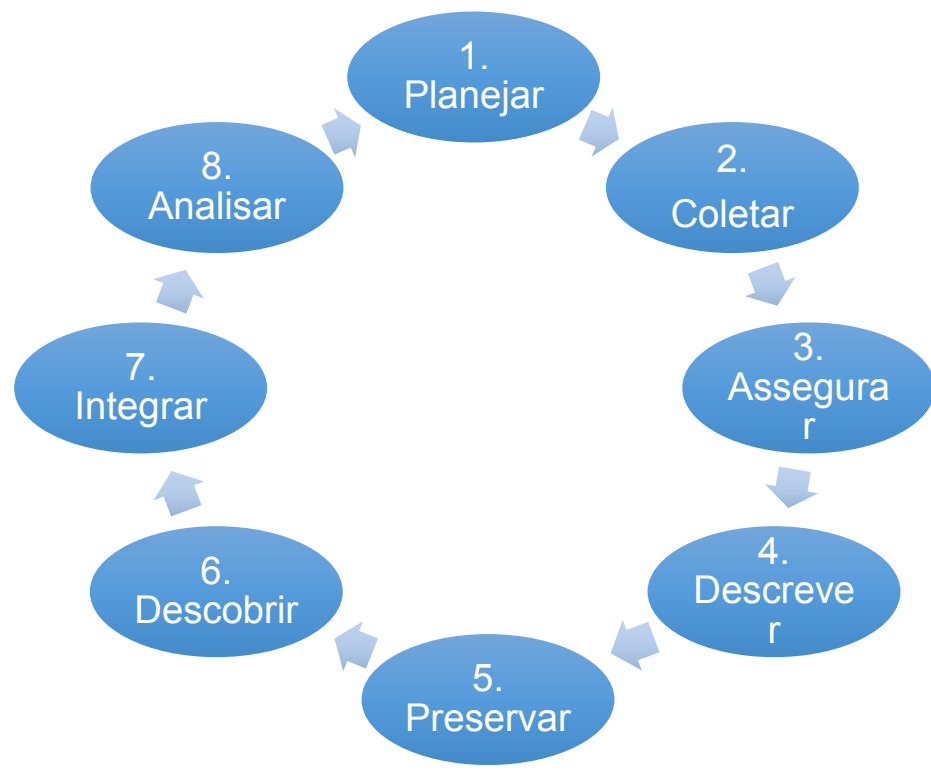

Fonte: Adaptação nossa (DataONE, online).

Recomenda-se a realização de alguns procedimentos que devem ser adotados para a efetivação de cada uma delas, descritos, a seguir.

A primeira etapa, 1. Planejar, corresponde a um prévio planejamento de como todo o processo vai ocorrer, ou seja, todos as etapas e procedimentos metodológicos devem ser pensados neste primeiro momento. Dentre as atividades aqui realizadas, destacamos o planejamento de como será realizada a coleta dos dados (quais dados serão gerados, como serão coletados e analisados, com qual método e instrumentos); onde os dados deverão ser armazenados (escolha de um repositório); como os dados serão organizados (em que formato, por qual tipo); como os dados serão gerenciados (definir o responsável pelos dados; como serão atualizados e com que frequência); como os dados serão descritos de forma a facilitar sua preservação e posterior acesso (se fará uso de metadados e padrão de metadados); como os conjuntos de dados serão compartilhados (se apenas entre a equipe do projeto e/ou com a comunidade científica; terá acesso aberto ou será embargado por determinado tempo e como serão divulgados); como vai se dar o processo de preservação desses dados (implementar plano de preservação, utilizar diversos locais de armazenamento; realizar atualização constante); e por fim, o orçamento deverá ser considerado no que concerne à equipe de apoio, recursos 
computacionais (STRASSER et al., 2012).

A segunda etapa, 2. Coletar, será onde os dados primários serão coletados, e como serão coletados. Para tal, recomenda-se: criar um modelo para coleta (visando apenas uma forma de coleta, caso haja uma equipe para coleta); descrever o conteúdo dos arquivos dos dados (através de parâmetros, formatos, unidades); organizar os dados em um arquivo (seja em planilhas ou banco de dados); usar o mesmo formato de dados em todo o arquivo (visando a padronização); usar caracteres de texto simples (visando a legibilidade do arquivo por um maior número de softwares); usar software e hardware em todo o processo de coleta (de preferências estáveis e não proprietários); atribuir nomes de arquivos descritivos (de movo a descrever o projeto, conteúdo do arquivo, data, localização); manter os dados brutos crus; criar tabelas de parâmetros (descrevendo códigos, abreviaturas, unidades, dado numérico, dado textual) (STRASSER et al., 2012).

A etapa 3. Assegurar, se dá por meio da adoção de critérios e procedimentos que venham assegurar a qualidade e validação dos dados, objetivando sua autenticidade e relevância, em todos os estágios e o gerenciamento a longo prazo (OLIVEIRA et al., 2015). Compreende a execução da garantia básica de qualidade e do controle de qualidade, por meio da descrição das condições durante a coleta (que ocasionalmente podem vir a afetar a qualidade dos dados); verificação dos dados (utilizar conjuntos de dados similares para identificar possíveis problemas); verificação daqueles dados que são manualmente inseridos, como do formato dos dados; e execução de resumos estatísticos e gráficos (de forma a verificar valores questionáveis, impossíveis ou faltantes) (STRASSER et al., 2012).

A quarta etapa, 4. Descrever, contempla a descrição minuciosa dos dados de pesquisa por meio da adoção de padrões e ferramentas de metadados, além de permitir a interoperabilidade por meio dos metadados, a qual permite que outros descubram, compreendam e usem esses dados (OLIVEIRA et al., 2015). Neste estágio é realizada a documentação completa dos dados (designada metadados), visto que descrever o contexto digital do conjunto de dados (nome do conjunto, nome dos arquivos do conjunto, data da última 
modificação, software utilizado); descreve o pessoal e as partes interessadas (descrição da equipe de coleta, responsável pelo conjunto de dados, patrocinadores da pesquisa); também descreve o contexto científico pelo qual os dados foram coletados (como foram coletados, o instrumentos usado); dar informações sobre os parâmetros (unidade de medida, formato do conjunto de dados, definição dos códigos usados), bem como, informar como o conjunto de dados deve ser citado (STRASSER et al., 2012).

No que se refere a etapa 5. Preservar, o plano de preservação dos dados em curto e longo prazo são traçados. Em curto prazo objetivando estocar e preservar a coleta primária dos dados. E em longo prazo visando possibilitar a reutilização desses dados no futuro pela comunidade científica (OLIVEIRA et al., 2015). Faz-se crucial ao estágio de preservar, o trabalho em conjunto com um centro/repositório de dados, os quais fornecem algumas orientações (como gerar os metadados, preservar os dados, quais formatos usar), e se configuram como uma ferramenta para descoberta, acesso e disseminação dos dados. Além de fazer-se necessário o uso de terminologias padrões (como palavras-chave, ontologias); políticas gerais (permissões passa usar dados, dados licenciados); atribuição e proveniência onde as seguintes informações devem ser incluídas: o pessoal responsável pelo conjunto de dados; o contexto do conjunto de dados; histórico de revisão; links para os dados de origem; como citar o conjunto de dados; direitos de propriedade intelectual (STRASSER et al., 2012).

A sexta etapa, 6. Descobrir, configura-se na identificação de outros conjuntos e dados e repositórios que possam complementar e agregar valor à determinada pesquisa (OLIVEIRA et al., 2015). Além de fazer com que "seus" dados também sejam utilizados em outras pesquisas, fundamentando-se na Lei de Boyle que "o futuro da ciência reside na compreensão do passado". Para isso, se fazem necessários a divulgação, o compartilhamento (promover a cultura de compartilhamento e reutilização de dados entre criadores de dados e usuários) e a publicação dos dados utilizando ferramentas e serviços da web garantindo que os conjunto de dados sejam reprodutíveis, além de, vistos e/ou acessados por qualquer pessoa (STRASSER et al., 2012).

A 7. Integrar, consiste na integração do "seu" conjunto de dados a outros 
recursos de dados que possam proporcionar novas análises e investigações, também tomando por base a Lei de Boyle mencionada anteriormente (OLIVEIRA et al., 2015). Para que haja a integração é necessário que os dados sejam compatíveis e comparáveis (compatibilidade dos dados); documente minuciosamente as etapas realizadas (permitindo que outros possam avaliar e interpretar seus resultados) como também a integração de vários conjuntos de dados (STRASSER et al., 2012).

Por fim, a última etapa, 8. Analisar, consiste na exploração, análise e visualização dos dados, e posteriormente, nas derivações dos seus dados através de publicações (OLIVEIRA et al., 2015). Para tanto, é preciso que os métodos utilizados (visuais e/ou estatísticos) e as etapas sejam documentadas, o software mais apropriado seja indicado, para que os dados sejam compatíveis, comparáveis, reprodutíveis.

É válido ressaltar que as etapas componentes do Ciclo de Vida dos Dados - DataONE não são dependentes entre si, e não são completamente obrigatórias para sua otimização. As etapas podem e devem ser realizadas de acordo com as necessidades de cada pesquisador, comunidade científica, entre outros.

\section{COMPETÊNCIAS DOS PROFISSIONAIS DA INFORMAÇÃO}

No que concerne às competências dos profissionais da informação, Abels et al. (2003), no relatório intitulado "Competências para profissionais da informação do século 21" apresentam que o profissional da informação é aquele que:

[...] utiliza informação em seu trabalho de forma a contribuir para que a organização cumpra sua missão. O profissional da informação realiza isso por meio do desenvolvimento, implementação e gerência de recursos e serviços de informação. O profissional da informação aproveita a tecnologia como uma ferramenta crítica para atingir metas ${ }^{8}$. (ABELS et al., 2003, p. 1, tradução nossa).

Para efeitos deste estudo, faremos uso das competências dos

8 [...] uses information in his/her job to advance the mission of the organization. The IP accomplishes this through the development, deployment, and management of information resources and services. The IP harnesses technology as a critical tool to accomplish goals. IPs include, but are not limited to librarians, knowledge managers, chief information officers, web developers, information brokers, and consultants. 
profissionais da informação propostas por Abels et al. (2003, tradução nossa), no qual afirmam que os profissionais da informação necessitam de competências pessoais e profissionais e declaram ser "absolutamente essenciais para todos os profissionais da informação".

No que concerne às competências pessoais, configuram-se como a união de atitudes, habilidades e valores, que vão permitir que os profissionais da informação tenham desempenho efetivo e contribuam de forma positiva em seus ambientes de trabalho (ABELS et al., 2003).

De acordo com Abels et al. (2003) as competências profissionais são divididas em quatro grupos, os quais serão descritos, no Quadro 1, a seguir:

Quadro 1 - Competências dos profissionais da informação

\begin{tabular}{|c|c|}
\hline \multicolumn{2}{|r|}{ COMPETÊNCIAS PROFISSIONAIS } \\
\hline $\begin{array}{c}\text { GRUPOS DE } \\
\text { COMPETÊNCIA }\end{array}$ & COMPETÊNCIAS \\
\hline $\begin{array}{l}\text { 1. GERENCIAR } \\
\text { ORGANIZAÇÕES } \\
\text { DE } \\
\text { INFORMAÇÃO }\end{array}$ & $\begin{array}{l}\text { 1.1 Alinha a organização da informação e apoia as estratégias da } \\
\text { organização, ou dos principais grupos de clientes através de } \\
\text { parcerias com os principais interessados e fornecedores. } \\
\text { 1.2 Avalia e comunica o valor da organização da informação, inclui } \\
\text { serviços de informação, produtos e políticas para } \\
\text { administração, partes interessadas e grupos de clientes. } \\
\text { 1.3 Estabelece processos eficazes de gestão, gestão operacional } \\
\text { e financeira. Faz julgamentos comerciais e financeiros sólidos } \\
\text { na tomada de decisões que equilibrem considerações } \\
\text { operacionais e estratégicas. } \\
\text { 1.4 Contribui efetivamente para as estratégias de gerenciamento } \\
\text { sênior e decisões sobre aplicações, ferramentas e tecnologias } \\
\text { de informação, e políticas para a organização. } \\
\text { 1.5 Desenvolve e lidera uma equipe efetiva de serviços de } \\
\text { informação e defende o desenvolvimento profissional e pessoal } \\
\text { das pessoas que trabalham na organização da informação. } \\
\text { 1.6 Mercados de serviços e produtos de informações, tanto } \\
\text { formalmente como informalmente, através de comunicação } \\
\text { física e web, apresentações, publicações e conversas. } \\
\text { 1.7 Reúne as melhores evidências disponíveis para apoiar } \\
\text { decisões sobre o desenvolvimento de novos serviços e } \\
\text { produtos, a modificação de serviços atuais ou a eliminação de } \\
\text { serviços para melhorar continuamente a variedade de serviços } \\
\text { de informação oferecidos. } \\
\text { 1.8 Aconselha a organização sobre direitos autorais e questões de } \\
\text { propriedade intelectual e conformidade. }\end{array}$ \\
\hline $\begin{array}{l}\text { 2. GERENCIAR } \\
\text { RECURSOS DE } \\
\text { INFORMAÇÃO }\end{array}$ & $\begin{array}{l}\text { 2.1 Gerencia o ciclo de vida completo da informação de sua criação } \\
\text { ou aquisição. Isso inclui a organização, categorização, } \\
\text { catalogação, classificação, disseminação. Criando e } \\
\text { gerenciamento taxonomias, tesauros, etc. }\end{array}$ \\
\hline
\end{tabular}

Inf. Inf., Londrina, v. 24, n. 1, p. 80 - 101, jan./abr. 2019. 


\begin{tabular}{|c|c|}
\hline & $\begin{array}{l}\text { 2.2 Desenvolve uma coleção dinâmica de recursos de informação } \\
\text { com base em uma compreensão profunda das necessidades } \\
\text { de informação dos clientes e seus processos de aprendizagem, } \\
\text { trabalho e/ou negócios. } \\
\text { 2.3 Demonstra conhecimento especializado sobre o conteúdo e o } \\
\text { formato dos recursos de informação, incluindo a capacidade de } \\
\text { avaliar, selecionar e filtrá-los criticamente. } \\
\text { 2.4 Fornece acesso aos melhores recursos de informações } \\
\text { disponíveis externamente e internamente, e implanta conteúdo } \\
\text { em toda a organização usando um conjunto de ferramentas de } \\
\text { acesso a informações. } \\
\text { 2.5 Negocia a compra e o licenciamento de produtos e serviços de } \\
\text { informações necessários. } \\
\text { 2.6 Desenvolve políticas de informação para a organização em } \\
\text { relação a recursos de informações, e aconselha sobre a } \\
\text { implementação dessas políticas. }\end{array}$ \\
\hline $\begin{array}{l}\text { 3. GERENCIAR } \\
\text { SERVIÇOS DE } \\
\text { INFORMAÇÃO }\end{array}$ & $\begin{array}{l}\text { 3.1 Desenvolve e mantém um portfólio de serviços de informação } \\
\text { que estão alinhados com as orientações estratégicas da } \\
\text { organização e dos grupos de clientes. } \\
\text { 3.2 Realiza pesquisas de mercado dos comportamentos de } \\
\text { informação e problemas de grupos de clientes atuais e } \\
\text { potenciais para identificar conceitos para soluções de } \\
\text { informação novas ou aprimoradas para esses grupos. } \\
\text { Transforma esses conceitos em produtos e serviços de } \\
\text { informações personalizados. } \\
\text { 3.3 Pesquisa, analisa e sintetiza informações em respostas } \\
\text { precisas ou informações acionáveis para clientes e garante que } \\
\text { os clientes tenham as ferramentas ou capacidades para aplicar } \\
\text { imediatamente essas. } \\
\text { 3.4 Desenvolve e aplica métricas adequadas para medir } \\
\text { continuamente a qualidade e o valor das ofertas de informações } \\
\text { e tomar as medidas adequadas para garantir a relevância de } \\
\text { cada oferta dentro do portfólio. } \\
\text { 3.5 Emprega gestão baseada em evidências para demonstrar o } \\
\text { valor e melhorar continuamente as fontes e serviços de } \\
\text { informação. }\end{array}$ \\
\hline $\begin{array}{l}\text { 4. APLICAÇÃO } \\
\text { DE } \\
\text { FERRAMENTAS } \\
\text { E } \\
\text { TECNOLOGIAS } \\
\text { DE } \\
\text { INFORMAÇÃO }\end{array}$ & $\begin{array}{l}\text { 4.1 Avalia, seleciona e aplica ferramentas de informação atuais e } \\
\text { emergentes e cria soluções de acesso e entrega de } \\
\text { informações. } \\
\text { 4.2 Aplica conhecimentos em bases de dados, indexação, } \\
\text { metadados e análise e síntese de informações para melhorar a } \\
\text { recuperação e utilização da informação na organização. } \\
\text { 4.3 Protege a privacidade das informações dos clientes e mantém } \\
\text { a consciência e as respostas aos novos desafios à privacidade. } \\
\text { 4.4 Mantém a consciência atual das tecnologias emergentes que } \\
\text { podem não ser atualmente relevantes, mas podem se tornar } \\
\text { ferramentas relevantes de recursos, serviços ou aplicativos de } \\
\text { informação futuros. }\end{array}$ \\
\hline
\end{tabular}

Fonte: Abels et al. (2003, tradução nossa).

De acordo com o que foi exposto, pode-se perceber que as competências dos profissionais da informação propostas por Abels et al. (2003), compreendem 
quatros grandes grupos, que englobam o gerenciamento de todos os elementos de uma organização informacional (recursos humanos, produtos e serviços e ferramentas).

\section{CICLO DE VIDA DOS DADOS E O PROFISSIONAL DA INFORMAÇÃO: CONVERGÊNCIAS E DIVERGÊNCIAS}

Para realizarmos o estudo comparado, selecionamos os grupos de competências propostos por Abels et al. (2003) dos profissionais da informação, bem como, as competências/atividades profissionais propriamente ditas e as relacionamos com as oito etapas componentes do Ciclo de Vida dos Dados DataONE.

De forma a facilitar o entendimento dos quadros comparativos, enumeramos os grupos das competências profissionais dos profissionais da informação, bem como, as atividades exercidas em cada um desses grupos, as quais iremos relacionar com as etapas componentes do Ciclo de Vida dos Dados - DataONE.

O primeiro grupo de competência "Gerenciar Organizações de Informação" bem como suas competências se correlacionam com as etapas do Ciclo de Vida de Dados - DataONE, conforme Quadro 2.

Quadro 2 - Gerenciar organizações de informação e o Ciclo de Vida dos Dados DataONE

\begin{tabular}{|c|c|c|}
\hline $\begin{array}{c}\text { GRUPO DE } \\
\text { COMPETÊNCIA }\end{array}$ & COMPETÊNCIAS & $\begin{array}{c}\text { ETAPAS Ciclo de Vida } \\
\text { dos Dados - DataONE }\end{array}$ \\
\hline \multirow{4}{*}{$\begin{array}{c}\text { 1. GERENCIAR } \\
\text { ORGANIZAÇÕES DE } \\
\text { INFORMAÇÃO }\end{array}$} & 1.1 & 1. Planejar \\
\cline { 2 - 3 } & 1.2 & 6. Descobrir \\
\cline { 2 - 3 } & 1.3 & 1. Planejar \\
\cline { 2 - 3 } & 1.4 & 1. Planejar \\
\cline { 2 - 3 } & 1.5 & 1. Planejar \\
\cline { 2 - 3 } & 1.6 & 1. Descobrir \\
\cline { 2 - 3 } & 1.7 & 2. Coletar \\
\cline { 2 - 3 } & & 6. Descobrir \\
\cline { 2 - 3 } & 1.8 & 7. Integrar \\
\cline { 2 - 3 } & & 3. Assegurar \\
\hline
\end{tabular}

Fonte: Elaboração nossa a partir dos dados da pesquisa, 2017. 
Como podemos perceber por meio do Quadro 2, a competência 1.1, trata sobre o alinhamento da organização da informação de forma a apoiar as estratégias da organização (unidade informacional), associando-se a etapa "1. Planejar" onde é realizado todo o planejamento prévio de como vai se dar o ciclo de vida dos dados.

A competência 1.2, comunica os serviços de informação para as partes interessadas, bem como, a etapa "6. Descobrir" que por meio da disseminação, envolve a descoberta e acesso aos dados úteis.

Na competência 1.3, o profissional da informação - abreviado para PI estabelece processos eficazes de gestão estando diretamente relacionada a primeira etapa do Ciclo de Vida dos Dados - DataONE, "Planejar".

A competência 1.4 também é diretamente relacionada a etapa do "1. Planejar" já que o $\mathrm{PI}$ deve contribuir com as estratégias de gerenciamentos. Como também, deve liderar uma equipe para trabalhar na organização da informação, competência 1.5, assim como deve "1. Planejar" as tarefas exercidas pelo pessoal de apoio ao ciclo de vida dos dados.

A competência 1.6 argumenta que através de canais formais ou informais de comunicação o $\mathrm{PI}$ deve divulgar os produtos e serviços de informação, o que se relaciona a etapa de descoberta dos dados que é realizada por meio da divulgação dos mesmos.

A competência 1.7 relaciona-se com o Ciclo de Vida dos Dados DataONE no sentido de que o PI deve apoiar as decisões da unidade informacional, e para isso, deve "1. Planejar"; "3. Coletar"; "6. Descobrir" e "7. Integrar" informações, assim como os dados, passam por estes processos para serem analisados, e (re)utilizados nos mais diversos fins.

Por fim, a competência 1.8 recomenda que o PI oriente a organização sobre questões de direito autoral, propriedade intelectual, o que é fundamental na realidade dos dados, e ao processo de "3. Assegurar" e "5. Preservar" do Ciclo de Vida dos Dados - DataONE.

Conforme apresentado na tabela, podemos perceber no que concerne ao primeiro grupo de competências dos profissionais da informação proposto por Abels et al. (2003) "Gerenciar organizações de informação" que às competências 
inerentes ao mesmo se relacionam com o Ciclo de Vida dos Dados - DataONE, já que o mesmo consiste em um processo de gestão de dados, traçando como os dados devem ser tratados em todos os estágios do seu ciclo de vida. $\mathrm{O}$ profissional da informação tem de estar apto a gerenciar as organizações de informação, assim como, há de estar apto a exercer e adequar suas competências na realidade da gestão dos dados durante todo o seu ciclo de vida.

O Quadro 3, correlaciona o segundo grupo de competências "Gerenciar Recursos de Informação" para com as etapas componentes do Ciclo de Vida dos Dados - DataONE.

Quadro 3 - Gerenciar recursos de informação e o Ciclo de Vida dos Dados - DataONE

\begin{tabular}{|c|c|c|}
\hline $\begin{array}{c}\text { GRUPO DE } \\
\text { COMPETÊNCIA }\end{array}$ & COMPETÊNCIAS & $\begin{array}{c}\text { ETAPAS } \\
\text { Ciclo de Vida dos } \\
\text { Dados - DataONE }\end{array}$ \\
\hline \multirow{11}{*}{$\begin{array}{l}\text { 2. GERENCIAR } \\
\text { RECURSOS DE } \\
\text { INFORMAÇÃO }\end{array}$} & 2.1 & 1. Planejar \\
\hline & \multirow{3}{*}{2.2} & 2. Coletar \\
\hline & & 6. Descobrir \\
\hline & & 7. Integrar \\
\hline & \multirow{2}{*}{2.3} & 3. Assegurar \\
\hline & & 4. Descrever \\
\hline & \multirow{2}{*}{2.4} & 5. Preservar \\
\hline & & 6. Descobrir \\
\hline & 2.5 & 7. Integrar \\
\hline & \multirow{2}{*}{2.6} & 3. Assegurar \\
\hline & & 5. Preservar \\
\hline
\end{tabular}

Fonte: Elaboração nossa a partir dos dados da pesquisa, 2017.

De acordo com o Quadro 3, o segundo grupo de competências "Gerenciar recursos de informação" a primeira competência 2.1 corresponde ao gerenciamento do ciclo de vida completo da informação estando completamente relacionada a etapa "1. Planejar" a qual gerencia previamente todo o processo do ciclo de vida dos dados.

A competência 2.2 recomenda ao $\mathrm{PI}$ o desenvolvimento de uma coleção de recursos de informação com bases nas necessidades informacionais dos usuários e da organização, estando relacionada as etapas de "2. Coletar"; "6. Descobrir" e "7. Integrar", visto que tratam sobre a coleta dos dados, a descoberta dos mesmos e integração deles em conjuntos homogêneos de dados necessários à determinada pesquisa. 
Na competência 2.3 o PI deve demonstrar conhecimento especializado no que concerne ao tratamento da informação, adequando-se à realidade do ciclo de vida dos dados em que as etapas de "3. Assegurar" e "4. Descrever" são etapas de processamento técnico inerente aos dados.

O PI deve fornecer acesso aos recursos de informações, competência 2.4, assim como deve fornecer acesso aos conjuntos de dados, por meio das etapas de preservação que vai garantir acesso, a longo prazo, por meio da descoberta.

A competência 2.5 adequa-se à etapa da integração dos dados homogêneos em conjuntos de dados, já que estes podem ser considerados produtos, podendo ser negociados, bem como, ter o acesso licenciado.

Por fim, a competência 2.6, recomenda que o PI desenvolva as políticas da organização, em relação aos recursos informacionais. Sendo esta prática também necessária a realidade dos dados de pesquisa, já que os mesmos precisam ser regidos sob considerações legais e políticas no que concerne seu uso e reuso.

O segundo grupo de competências profissionais "Gerenciar recursos de informação" trata do recurso informacional, da informação como um produto. $\mathrm{E}$ relaciona-se com o Ciclo de Vida dos Dados - DataONE, visto que o mesmo também trata do dado como sendo uma informação bruta, assegurando sua qualidade, por meio de tomada de decisões para que esta qualidade seja preservada a longo prazo, de modo que formem conjuntos de dados brutos a partir da sua disseminação, e que posteriormente serão (re) utilizados em outras investigações científicas.

O Quadro 4, relaciona o terceiro grupo de competências "Gerenciar Serviços de Informação" para com as etapas componentes do Ciclo de Vida dos Dados - DataONE.

Quadro 4 - Gerenciar serviços de informação e o Ciclo de Vida dos Dados DataONE

\begin{tabular}{|c|c|c|}
\hline $\begin{array}{c}\text { GRUPO DE } \\
\text { COMPETÊNCIA }\end{array}$ & COMPETÊNCIAS & $\begin{array}{c}\text { ETAPAS Ciclo de Vida } \\
\text { dos Dados - DataONE }\end{array}$ \\
\hline $\begin{array}{c}\text { 3. GERENCIAR } \\
\text { SERVIÇOS DE } \\
\text { INFORMAÇÃO }\end{array}$ & 3.1 & 2. Coletar \\
\cline { 2 - 3 } & 3.2 & 6. Descobrir \\
\hline
\end{tabular}

Inf. Inf., Londrina, v. 24, n. 1, p. 80 - 101, jan./abr. 2019. 


\begin{tabular}{|c|c|c|}
\hline \multirow{4}{*}{} & & 6. Descobrir \\
\cline { 2 - 3 } & \multirow{3}{*}{3.3} & 2. Coletar \\
\cline { 2 - 3 } & & 7. Integrar \\
\cline { 2 - 3 } & 3.4 & 8. Analisar \\
\cline { 2 - 3 } & \multirow{3}{*}{3.5} & 3. Assegurar \\
\cline { 2 - 3 } & & 3. Planejar \\
\cline { 2 - 3 } & & 3. Assegurar \\
\hline
\end{tabular}

Fonte: Elaboração nossa a partir dos dados da pesquisa, 2017.

Com base no estudo comparado apresentado no Quadro 4, a primeira competência, 3.1, concerne na elaboração de um portfólio dos serviços de informação que são alinhados com as estratégias da organização. E é passível de adaptação à etapa "2. Coletar" visto que os serviços de informação precisam ser selecionados, e integrados visando a elaboração do portfólio, bem como, os dados precisam ser coletados no para dar continuidade ao processo do ciclo de vida dos dados.

A competência 3.2 também se adequa à realidade do ciclo de vida dos dados, já que o $\mathrm{PI}$ deve transformar as informações, em produtos oferecedores de serviços, bem como, os dados. Já que os mesmos se configuram como fonte primária de informação, sendo coletados como produto, e descobertos por serviços especializados para o seu posterior (re)uso.

$\mathrm{Na}$ competência 3.3 o PI deve pesquisar (2. Coletar), sintetizar (7. Integrar) e analisar as informações de acordo com as necessidades informacionais de seus usuários. Assim como acontece no Ciclo de Vida dos Dados - DataONE, onde os PI precisam auxiliar a comunidade de pesquisadores nestes mesmos processos, em sendo o objeto de estudo, os seus dados de pesquisa.

O PI deve medir continuamente a qualidade das informações que ele disponibiliza, o que corresponde a competência 3.4, assim como os dados devem ter sua qualidade assegurada por meio de controles de inspeções de modo a verificar valores questionáveis e consequentemente se tornarem confiáveis.

A competência 3.5 argumenta que os serviços de informação devem ser continuamente melhorados, desta forma, faz-se necessário uma gestão 
recorrente. Assim como o ciclo de vida dos dados, é preciso que o mesmo seja atualizado de acordo com o decorrer e com as necessidades da pesquisa.

No que concerne ao terceiro grupo de competências "Gerenciar serviços de informação", a informação é tida como um produto que demanda por serviços. Assim como os dados. Em conformidade com a informação em seu fluxo informacional, os dados também precisam ser 'tecnicamente' tratados durante todo o seu ciclo de vida, e faz-se necessário a capacitação ou adaptação/capacitação dos profissionais da informação para estes fins.

O Quadro 5, relaciona o quarto grupo de competências profissionais "Aplicar ferramentas e tecnologias da informação" para com as etapas componentes do Ciclo de Vida dos Dados - DataONE.

\section{Quadro 5 - Aplicar Ferramentas e Tecnologias de Informação e o Ciclo de Vida} dos Dados - DataONE

\begin{tabular}{|c|c|c|}
\hline $\begin{array}{c}\text { GRUPO DE } \\
\text { COMPETENCIA }\end{array}$ & COMPETÊNCIAS & $\begin{array}{c}\text { ETAPAS } \\
\text { Ciclo de Vida dos Dados } \\
\text { - DataONE }\end{array}$ \\
\hline \multirow{12}{*}{$\begin{array}{l}\text { 4. APLICAR } \\
\text { FERRAMENTAS E } \\
\text { TECNOLOGIAS DE } \\
\text { INFORMAÇÃO }\end{array}$} & \multirow{4}{*}{4.1} & 2. Coletar \\
\hline & & 5. Preservar \\
\hline & & 7. Integrar \\
\hline & & 8. Analisar \\
\hline & \multirow{5}{*}{4.2} & 2. Coletar \\
\hline & & 3. Assegurar \\
\hline & & 4. Descrever \\
\hline & & 5. Preservar \\
\hline & & 8. Analisar \\
\hline & \multirow{2}{*}{4.3} & 3. Assegurar \\
\hline & & 5. Preservar \\
\hline & 4.4 & - \\
\hline
\end{tabular}

Fonte: Elaboração nossa a partir dos dados da pesquisa, 2017.

No que concerne a competência 4.1, a mesma argumenta que o PI deve avaliar, selecionar e aplicar ferramentas de informação para o acesso às mesmas. Assim como no Ciclo de Vida dos Dados - DataONE, onde os dados são coletados, preservados, por meio de ferramentas, integrados em conjuntos homogêneos de dados para posterior acesso, e análise/uso.

A competência 4.2 recomenda que o $\mathrm{Pl}$ aplique o seu conhecimento em indexação, metadados de forma a melhorar a recuperação e utilização da 
informação. Estando diretamente relacionada ao ciclo de vida dos dados, no qual, após a coleta dos dados, os mesmo têm sua qualidade assegurada, são descritos por meio de metadados e preservados em repositórios de forma a garantir a sua recuperação, o seu acesso e posterior uso por meio da análise.

Na competência 4.3 o PI é responsável por proteger as informações em relação às questões de privacidade, como acontece com os dados, e as etapas de "3. Assegurar" e "5. Preservar" que consistem na atribuição das políticas de privacidade, direito autoral, e propriedade intelectual.

A competência 4.4 versa que os $\mathrm{PI}$ devem estar sempre atentos às tecnologias emergentes, já que as mesmas podem vir a tornar-se ferramentas relevantes e atuantes para os serviços e procedimentos futuros. Assim como em todo o ciclo de vida dos dados, as inúmeras tecnologias da informação, podem auxiliar em todos as etapas componentes do Ciclo de Vida dos Dados DataONE.

Pode-se perceber que o último grupo de competências "Aplicar ferramentas e tecnologias da informação" em síntese, confirma que as tecnologias da informação se configuram como ferramentas indispensáveis no que concerne ao tratamento da informação como um todo. Concomitantemente as Tecnologias de Informação (Tls) também se configuram como uma ferramenta indispensável e de alta contribuição no processo do ciclo de vida dos dados.

\section{CONSIDERAÇÕES FINAIS}

Com a análise referente as competências dos profissionais da informação propostas por Abels et al. (2003), percebe-se que três dos quatros grupos de competências refere-se ao gerenciamento da organização como unidade informacional, com seus recursos humanos, produtos e serviços, em relação às estratégias, valores, políticas da organização superior da qual faz parte. Em sintonia com o ciclo de vida dos dados o qual é anteriormente planejado e alinhado de acordo com os objetivos propostos de determinada pesquisa. Fazendo com que o profissional da informação possa atuar de forma satisfatória em ambos cenários fazendo uso das suas competências profissionais. 
O quarto grupo de competências, refere-se à aplicação de ferramentas e tecnologias da informação, o qual se encontra amplamente relacionado à realidade da e-Science, mais precisamente ao ciclo de vida dos dados, visto que em todas as componentes do ciclo de vida dos dados, o uso da tecnologia da informação apresenta-se de forma necessária e indispensável, visto que a própria e-Science originou-se neste cenário de ascensão das TDICs.

Concluímos que as competências dos profissionais da informação propostas por Abels et al. (2003), onde considerou a informação como objeto de estudo, podem ser efetivamente aplicadas e/ou adaptadas à realidade dos dados científicos, mais precisamente no processo do ciclo de vida dos dados, já que os mesmos se configuram como uma fonte primária de informação.

\section{REFERÊNCIAS}

ABELS, E. et al. Competencies for information professionals of the $\mathbf{2 1}$ century. 2003. Disponível em:

https://www.sla.org/wpcontent/uploads/2013/01/0_LRNCompetencies2003_revi sed.pdf. Acesso em: 23 jun. 2017.

BORGMAN, C. L. Big data, little data, no data: Scholarship in the Networked World. London: MIT Press, 2015.

BURTON, M.; LYON, L. Data Science in Libraries. Bulletin of the Association for Information Science and Technology, v. 43, n. 4, 2017. Disponível em: http://onlinelibrary.wiley.com/doi/10.1002/bul2.2017.1720430409/full. Acesso em: 23 jun. 2017.

CONEGLIAN, C. S.; SEGUNDO, J. E. S.; SANT'ANA, R. C. S. G. A. Big data: fatores potencialmente discriminatórios em análise de dados. Em Questão, v. 23, n. 1, 2017. Disponível em: http://www.brapci.ufpr.br/brapci/v/a/21379. Acesso em: 23 jun. 2017.

COSTA, M. M.; CUNHA, M. B. O bibliotecário no tratamento de dados oriundos da e-science: considerações iniciais. Perspectivas em Ciência da Informação, Belo Horizonte, v. 19, n. 3, p. 189-206, jul./set. 2014. Disponível em: http://www.scielo.br/pdf/pci/v19n3/a10v19n3.pdf. Acesso em: 23 jun. 2017.

DataONE. What is DataONE? Online. Disponível em:

https://www.dataone.org/what-dataone. Acesso em: 23 jun. 2017.

MARCONI, M. de A.; LAKATOS, E. M. Metodologia científica. 3. ed. São Paulo: Atlas, 2000.

Inf. Inf., Londrina, v. 24, n. 1, p. 80 - 101, jan./abr. 2019. 
OLIVEIRA, A. C. S. de; DIAS, G. A.; CORRÊA, P. L. P.; ANJOS, R. L. dos; SOUZA, V. M. de. Quarto paradigma científico: adoção do data life cycle model para o gerenciamento de dados científicos abertos do experimento e-quilt prototype. In: Conferência Luso-Brasileira (CONFOA), 6., 2015, Salvador. Anais eletrônicos... Salvador: UFB, 2015. Disponível em: https://drive.google.com/drive/folders/0B2CiJWPT1NhwcmNiZ1BwZWY5TVU. Acesso em: 20 jun. 2017.

MCAFEE, A.; BRYNJOLFSSON, E. Big Data: the management revolution. Harvard Business Review, Brighton, v. 90, n. 10, p. 61-67, out. 2012. Disponível em: https://hbr.org/2012/10/big-data-the-management-revolution\#. Acesso em: 22 jun. 2017.

SANT'ANA, R. C. G. Ciclo de vida dos dados: uma perspectiva a partir da ciência da informação. Informação \& Informação, Londrina, v. 21, n. 2, p. 116142, maio/ago. 2016. Disponível em:

http://www.uel.br/revistas/uel/index.php/informacao/article/view/27940/20124. Acesso em: 10 ago. 2017.

SALES, L. F.; SAYÃO, L. F. Há futuro para as bibliotecas de pesquisa no ambiente de e-Science? Informação \&Tecnologia (ITEC), v .2, n. 1, p. 30-52, jan./jul. 2015. Disponível em: http://periodicos.ufpb.br/ojs/index.php/itec/article/view/26029/14677. Acesso em: 23 jun. 2017.

SALES, L. F.; SAYÃO, L. F.; SOUZA, R. F. Publicações ampliadas: um novo modelo de publicação acadêmica para o ambiente de e-Science. In: Encontro Nacional de Pesquisa em Ciência da Informação (ENANCIB), 14., 2013, Florianópolis. Anais eletrônicos... Florianópolis: ENANCIB, 2013. Disponível em: http://eprints.rclis.org/20666/1/APRESENTA\%C3\%87\%C3\%830\%20\%20ENANCIB\%202013.pdf. Acesso em: 23 jun. 2017.

SAYÃO, L. F. SALES, L. F. Curadoria geral: um novo patamar para a preservação de dados digitais de pesquisa. Informação \& Sociedade:

Estudos, João Pessoa, v. 22, n. 3, p. 179-191, set./dez. 2012. Disponível em: http://www.ies.ufpb.br/ojs/index.php/ies/article/viewFile/12224/8586. Acesso em: 23 jun. 2017.

SAYÃO, L. F.; SALES, L. F. Dados de pesquisa: contribuição para o estabelecimento de um modelo de curadoria digital para o país. Tendências da Pesquisa Brasileira em Ciência da Informação, v. 6, n. 1, 2013. Disponível em: http://www.brapci.ufpr.br/brapci/v/a/14157. Acesso em: 01 ago. 2017.

STRASSER, C.; COOK, R.; MICHENER, W.; BUDDEN, A. Primer on Data Management: What you always wanted to know. Califórnia: CDL, 2012. Disponível em:

http://escholarship.org/uc/item/7tf5q7n3\#page-1. Acesso em: 12 jul. 2017. 
THE ECONOMIST. The world's most valuable resource is no longer oil, but data. 2017. Disponível em:

http://http://www.economist.com/news/leaders/21721656-data-economydemands-new-approach-antitrust-rules-worlds-most-valuable-resource. Acesso em: 23 jun. 2017.

\title{
INFORMATION PROFESSIONALS PERFORMANCE IN THE DATA LIFE CYCLE - DATAONE: A COMPARED STUDY
}

\begin{abstract}
Introduction: with the definitive consolidation of the Information and Communication Digital Technologies in all human processes in the beginning of the $21^{\text {st }}$ century, daily, it is produced an endless amount of data. This avalanche of data is known as the Big Data phenomenon. In the academic field, new possibilities related to the use of research data are emerging. Researchers from several different areas are paying special attention to the Data Management Plan, which involves the curatorship of the research data during all its life cycle, since the planning stage until its (re)use. Objective: aims to investigate how the information professionals can act on the component stages of the Data Life Cycle - DataONE. Methodology: is an exploratory-descriptive and bibliographical research, assuming the comparative method which associates the competences of the information professionals that can be applied and / or adapted in the accomplishment of the component stages of the data life cycle - DataONE. Results: it has been found that most of the skills of information professionals are aligned with the components of the data life cycle. Conclusions: it was possible to conclude that the competence of the information professionals can be effectively applied and suited to the reality of the research data, more precisely, in the process of the data life cycle.
\end{abstract}

Descriptors: Scientific data. Information professionals. Data life cycle. DataONE.

\section{ACTUACIÓN DE LOS PROFESIONALES DE LA INFORMACIÓN EN EL CICLO DE VIDA DE LOS DATOS - DATAONE: UN ESTUDIO COMPARADO}

\begin{abstract}
RESUMEN
Introducción: con la consolidación de las Tecnologías Digitales de Información y Comunicación en la vasta mayoría de los procesos humanos a principios del siglo XXI, diariamente, producimos una infinidad de datos. En el campo académico, nuevas posibilidades relacionadas al uso de datos de investigación, están emergiendo. Los investigadores de las más variadas áreas están prestando especial atención al Plan de Gestión de Datos, el cual involucra la curaduría de los datos de investigación, durante todo su ciclo de vida, desde su planificación hasta su (re) uso. Objetivo: busca investigar cómo los profesionales de la información pueden actuar en las etapas componentes del Ciclo de vida de los datos propuesto por el DataONE. Metodología: investigación
\end{abstract}

Inf. Inf., Londrina, v. 24, n. 1, p. 80 - 101, jan./abr. 2019. 
exploratoria-descriptiva, y bibliográfica, asumiendo el método comparado el cual asoció las competencias de los profesionales de la información que pueden ser aplicadas y / o adaptadas en la realización de las etapas componentes del Ciclo de vida de los datos DataONE. Resultados: se comprobó que la mayoría de las competencias de los profesionales de la información se alinean con los componentes del ciclo de vida de los datos. Conclusiones: Concluimos que las competencias de los profesionales de la información pueden ser efectivamente aplicadas y adecuadas a la realidad de los datos de investigación, más precisamente en el proceso del ciclo de vida de los datos.

Descriptores: Datos científicos. Professional de la información. Ciclo de vida de los datos. DataONE. 